\title{
Inhaled nitric oxide augments nitric oxide transport on sickle cell hemoglobin without affecting oxygen affinity
}

\author{
Mark T. Gladwin, ${ }^{1}$ Alan N. Schechter, ${ }^{2}$ James H. Shelhamer, ${ }^{1}$ Lewis K. Pannell, ${ }^{3}$ \\ Deirdre A. Conway, ${ }^{3}$ Borys W. Hrinczenko, ${ }^{2}$ James S. Nichols, ${ }^{1}$ Margaret E. Pease-Fye, ${ }^{1}$ \\ Constance T. Noguchi, ${ }^{2}$ Griffin P. Rodgers, ${ }^{4}$ and Frederick P. Ognibene ${ }^{1}$ \\ ${ }^{1}$ Critical Care Medicine Department, Warren G. Magnuson Clinical Center, National Institutes of Health, \\ Bethesda, Maryland 20892, USA \\ ${ }^{2}$ Laboratory of Chemical Biology, \\ ${ }^{3}$ Structural Mass Spectrometry Group, and \\ ${ }^{4}$ Molecular and Clinical Hematology Branch, National Institute of Diabetes, Digestive, and Kidney Diseases, \\ National Institutes of Health, Bethesda, Maryland 20892, USA
}

Address correspondence to: Mark T. Gladwin, Warren G. Magnuson Clinical Center, Critical Care Medicine Department, Building 10, Room 7D43, 10 Center Drive, MSC 1662, Bethesda, Maryland 20892-1662, USA.

Phone: (301) 496-9320; Fax: (301) 402-1213; E-mail: mgladwin@nih.gov.

Received for publication June 21, 1999, and accepted in revised form August 13, 1999.

Nitric oxide (NO) inhalation has been reported to increase the oxygen affinity of sickle cell erythrocytes. Also, proposed allosteric mechanisms for hemoglobin, based on S-nitrosation of $\beta$-chain cysteine 93 , raise the possibilty of altering the pathophysiology of sickle cell disease by inhibiting polymerization or by increasing NO delivery to the tissue. We studied the effects of a 2 -hour treatment, using varying concentrations of inhaled NO. Oxygen affinity, as measured by $\mathrm{P}_{50}$, did not respond to inhaled NO, either in controls or in individuals with sickle cell disease. At baseline, the arterial and venous levels of nitrosylated hemoglobin were not significantly different, but NO inhalation led to a dose-dependent increase in mean nitrosylated hemoglobin, and at the highest dosage, a significant arterial-venous difference emerged. The levels of nitrosylated hemoglobin are too low to affect overall hemoglobin oxygen affinity, but augmented NO transport to the microvasculature seems a promising strategy for improving microvascular perfusion.

J. Clin. Invest. 104:937-945 (1999).

\section{Introduction}

In sickle cell anemia erythrocytes, deoxygenation of sickle hemoglobin (hemoglobin S) leads to intracellular polymer formation, ultimately distorting the erythrocyte and retarding red cell transit through the microvasculature (1). Within the red cell, hemoglobin $S$ has reduced affinity for oxygen, compared with hemoglobin A, because of competition between polymerization and oxygen binding. This reduced oxygen affinity results in an increase in the partial pressure of oxygen necessary for $50 \%$ hemoglobin saturation $\left(\mathrm{P}_{50}\right)$. The $\mathrm{P}_{50}$ value is inversely related to the hemoglobin affinity for oxygen. A variety of therapeutic strategies for sickle cell disease aimed at reducing hemoglobin S polymerization have been under development in recent years. These strategies include attempts to modify the structure of hemoglobin $S$ to reduce the hydrophobic interactions between hemoglobin $\beta$ chains or to increase the affinity for oxygen, as well as attempts to decrease the concentration of hemoglobin $\mathrm{S}$ by increasing the percentage of fetal hemoglobin or by increasing red cell volume (2).

Nitric oxide (NO) has been recognized in the last decade to have important and diverse physiological effects. NO is synthesized in multiple cell types and tis- sues from the substrate L-arginine by NO synthase enzyme systems, which may be constitutive or inducible in response to physiological stimuli. In physiological solution, NO reacts rapidly with oxygen, heme groups, and various functional groups on biologic molecules, including thiols. In fact, the covalent formation of $S$ nitrosothiol compounds such as $S$-nitrosoglutathione and $S$-nitrosoalbumin in the lung airways, plasma, and other tissues may serve to stabilize this highly reactive molecule and deliver it to target sites of action (3-5).

Recent work by Stamler and colleagues suggests that NO, produced in the lungs, binds to the highly conserved cysteine residue 93 on the $\beta$ chain of oxyhemoglobin to form $S$-nitrosohemoglobin (SNOhemoglobin). NO thus carried on hemoglobin is envisioned to be transported to the microvasculature and may be released upon deoxygenation of the red cells in the tissues, resulting in vasodilation of the microvasculature (6-8). Recently, NO has also been reported to increase oxygen affinity of sickle cell erythrocytes (9). This effect was found to be dosedependent in vitro, with a maximum effect occurring at a dose of 80 parts per million (ppm). This effect was also apparent in vivo after delivery of $80 \mathrm{ppm} \mathrm{NO}$ for 
45 minutes. $P_{50}$ was reduced by an average of $4.6 \pm 2.0$ $\mathrm{mmHg}$ in patients with sickle cell anemia. These effects were specific for sickle red cells and were not associated with marked increases in methemoglobin formation. The mechanism of the reported hemoglobin S-specific increase in oxygen affinity, which might be of therapeutic value, is not certain.

To test the potential effects of NO on the structure and function of normal and sickle hemoglobin, we studied the effects of a 2-hour treatment of $80 \mathrm{ppm}$ inhaled $\mathrm{NO}$ (at a fraction of inspired oxygen $\left[\mathrm{FiO}_{2}\right]$ of 0.21 ) on normal (hemoglobin A genotype [AA]) and sickle cell (hemoglobin S genotype [SS]) individuals. We measured effects on NO metabolism, methemoglobin formation, hemoglobin oxygen affinity, and binding of NO to the hemoglobin molecule.

The term nitrosylated hemoglobin is used herein to represent hemoglobin with a bound NO molecule, either on the sulfhydryl of $\beta$-globin chain system 93 or on the heme iron.

\section{Methods}

Subjects. All protocols were approved by the National Heart, Lung, and Blood Institute's Institutional Review Board. All subjects signed an informed consent. Seven clinically stable volunteers with sickle cell disease (SS) and 5 normal volunteers (AA) were selected for study (Table 1). All volunteers had hemoglobin electrophoresis to confirm hemoglobin S or A phenotype, as well as hemoglobin F levels. Inclusion criteria for the individuals with sickle cell were as follows: age between 18 and 65 years, electrophoretic diagnosis of sickle cell disease, and a hematocrit greater than $18 \%$. Potential SS volunteers were excluded if they were clinically unstable, defined by having more than 2 pain crises that resulted either in a visit to the emergency department or in hospitalization during the preceding 2 months; undergoing therapy with hydroxyurea or butyrate any time in the preceding 12 months; being a smoker; or receiving blood transfusions within the preceding 3 months (or hemoglobin A >20\%).

$N O$ inhalation in SS and AA volunteers. Volunteers were instructed not to eat after midnight, and were given only water (orally) during the 6-hour study duration. A peripheral intravenous and radial artery catheter was placed. Venous and arterial blood samples were collected hourly; totals did not exceed $100 \mathrm{~mL}$ in a study day. Continuous blood pressure by cuff or arterial line, pulse oximetry, respiratory rate, temperature, and heart rate and rhythm were recorded. After an hour of baseline measurements, subjects breathed $\mathrm{NO}$ gas at a concentration of $80 \mathrm{ppm}$ (INOvent Delivery System; Datex-Ohmeda Inc., Madison, Wisconsin, USA). A room air gas condenser with an oxygen blender was used to deliver an $\mathrm{FiO}_{2}$ of 0.21 at $40 \mathrm{~L} / \mathrm{min}$ with an inline reservoir bag. $\mathrm{NO}$ gas was delivered in-line, and concentrations of $\mathrm{NO}$, oxygen, and nitrogen dioxide $\left(\mathrm{NO}_{2}\right)$ were sampled at the mask to ensure a delivery of $80 \mathrm{ppm} \mathrm{NO}$, an $\mathrm{FiO}_{2}$ of 0.21 , and to maintain the $\mathrm{NO}_{2}$

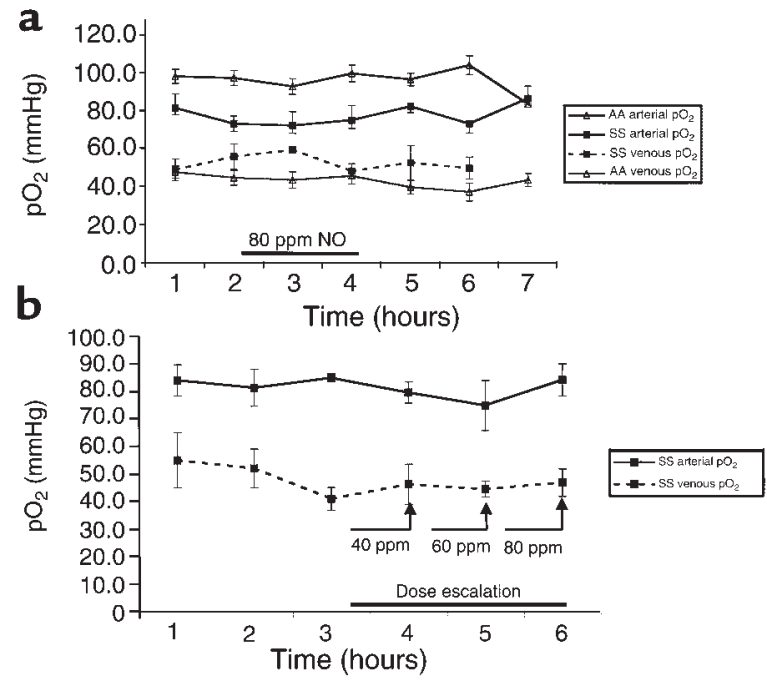

Figure 1

Effect of inhaled $\mathrm{NO}$ on arterial and venous $\mathrm{pO}_{2}$ in $\mathrm{SS}$ and $\mathrm{AA}$ individuals. (a) Before $\mathrm{NO}$ inhalation (hours 1 and 2), the $\mathrm{SS}$ arterial $\mathrm{pO}_{2}$ is lower than the $A A$ arterial $\mathrm{pO}_{2}$. The $\mathrm{SS}$ venous $\mathrm{pO}_{2}$ values are similar to the AA venous $\mathrm{pO}_{2}$ values, reflecting the reduced oxygen extraction or arterialization of venous blood that is characteristic of patients with sickle cell anemia. During 2 hours of 80 ppm NO inhalation (hours 3 and 4; filled horizontal bar), there is no significant change in $\mathrm{AA}$ or $\mathrm{SS}_{2} \mathrm{O}_{2}$ for arterial and venous blood. (b) During dose escalation of inhaled NO in $3 \mathrm{SS}$ individuals, there is no significant change in arterial or venous $\mathrm{pO}_{2}$ during 1-hour inhalation of 40, 60, and 80 ppm NO gas.

level below $1.0 \mathrm{ppm}$. With this system, inhaled NO concentrations remained constant at extremes of minute ventilation (data not shown). The study was conducted in a negative-flow isolation room, and no further NO scavenging was required, as room NO levels remained at less than 200 parts per billion (ppb) during treatment (measured with the Model 280 NO Analyzer; Sievers Instruments, Boulder, Colorado, USA). The NO was discontinued after 2 hours, and room NO levels dropped to less than $20 \mathrm{ppb}$ within 10 minutes. Blood was collected hourly for 3 more hours, at which time the study was terminated. In 3 additional SS patients, after 3 baseline blood collections, inhaled NO was delivered for 1 hour at $40 \mathrm{ppm}, 1$ hour at $60 \mathrm{ppm}$, and 1 hour at $80 \mathrm{ppm}$.

Oxygen dissociation curves. Oxygen dissociation curves (ODCs) were obtained using the Hemox-Analyzer (TCS Scientific Corp., New Hope, Pennsylvania, USA). During studies, the Hemox-Analyzer was located at the bedside of the volunteers. A total of $60 \mu \mathrm{L}$ of whole blood for SS individuals and $30 \mu \mathrm{L}$ of whole blood for AA individuals was added to $3 \mathrm{~mL}$ of buffer $(135 \mathrm{mM}$ $\mathrm{NaCl}, 30 \mathrm{mM}$ TES, $5 \mathrm{mM} \mathrm{KCl}$, and $\mathrm{NaOH}$ adjusted to pH $7.4 \pm 0.02$ [TCS buffer; TCS Scientific Corp.]), 7.5 $\mu \mathrm{L}$ of antifoam solution, and $15 \mu \mathrm{L}$ of $20 \%$ BSA. Samples were analyzed immediately upon collection from the patient. Nitrogen (100\%) was bubbled through the sample at a constant rate that resulted in complete deoxygenation within 20 minutes, followed by reoxy- 
genation with air for 15 minutes. The analyzer measured the oxygen tension with a standard Clark $\mathrm{O}_{2}$ electrode (Model 5331 Oxygen Probe; Yellow Springs Instrument Co., Yellow Springs, Ohio, USA) and simultaneously calculated the hemoglobin saturation using dual-wavelength spectrophotometry $(9,10)$. The ODCs were recorded during both deoxygenation and reoxygenation. During the study day, 7 hourly measurements of the deoxygenation and reoxygenation curves were obtained before, during, and after $\mathrm{NO}$ administration. To ensure reliable data, the oxygen electrode was cleaned and a new membrane applied 24 hours before each study day. The membrane was deoxygenated and reoxygenated in distilled water for a 12-hour period the day before all patient studies. The night before each study day, a sample of fresh AA blood was run as a standardization control.

Processing of blood. Arterial and venous blood samples were drawn, immediately protected from light, and centrifuged at $750 \mathrm{~g}$ for 5 minutes at room temperature. Plasma was removed and stored at $-70^{\circ} \mathrm{C}$ until assayed for nitrate/nitrite. The red blood cell pellet was removed and washed 2 times in 5 volumes of PBS. Cells were then lysed in a 1:4 dilution of $0.5 \mathrm{mM}$ EDTA in nitrite-free molecular biology grade water (Biofluids Inc., Rockville, Maryland, USA). After lysis, $500 \mu \mathrm{L}$ was passed through a $9.5-\mathrm{mL}$ bed volume Sephadex G25 column to remove nitrite and small thiols. The hemoglobin concentration of the Sephadex G25 effluent was measured by conversion to cyanomethemoglobin ( $\varepsilon_{540}=11$ for heme). (Note that all stated hemoglobin concentrations are in terms of heme.) Hemoglobin samples $(200 \mu \mathrm{L})$ were immediately drawn into $250-\mu \mathrm{L}$ Hamilton syringes and reacted with $\mathrm{I}_{3}{ }^{-}$. All measurements were performed within an hour of sample collection.

Determination of nitrate/nitrite using the Griess reaction. Serum samples were thawed, diluted 1:2 in HPLC-grade water, and filtered through a prewashed 30,000-molecular weight cutoff filtration unit (catalog no. UFC3LTKNB; Millipore Corp., Bedford, Massachusetts, USA). Samples and standards were reacted with and without nitrate reductase, according to the standard Griess reaction (11). In brief, nitrate present in samples is converted to nitrite using nitrate reductase, followed by stoichiometric reaction of nitrite with sulfanilamide and then $N$-(1-naphthyl)-ethylenediamine to form a purple azo product. The concentration of the azo product is measured in a spectrophotometer at a wavelength of $570 \mathrm{nM}$.

HPLC-electrospray ionization mass spectrometry. Mass spectrometry measurements of synthesized SNO-hemoglobin were performed to establish the specificity of the NO reaction with cysteine 93 and to test the operating characteristics of the $\mathrm{I}_{3}{ }^{-}$chemiluminescent technique (described later here). Patient samples were also run immediately upon collection to evaluate potential modification of the hemoglobin $\beta$ chain with NO, glutathione, or other low-molecular-weight adducts. Liquid chromatography mass spectrometry was performed
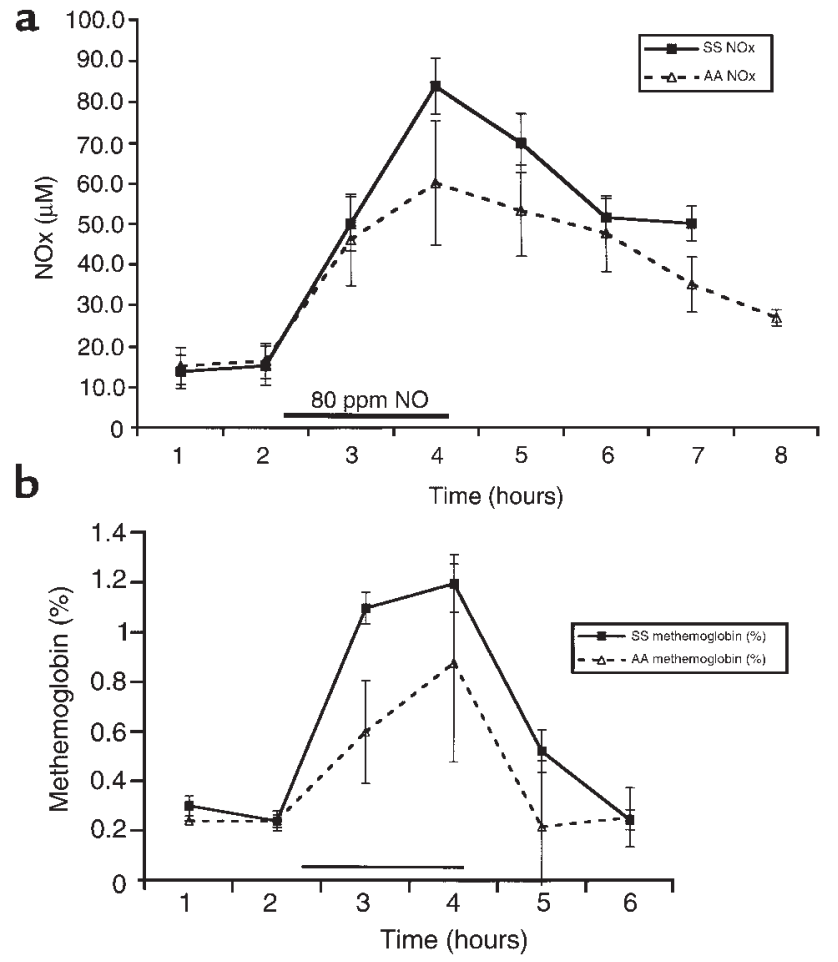

Figure 2

Effect of inhaled $80 \mathrm{ppm}$ inhaled $\mathrm{NO}$ on plasma $\mathrm{NO}_{x}$ (nitrate and nitrite measured by the Griess reaction) and methemoglobin. (a) For 2 hours before $\mathrm{NO}$ inhalation, mean plasma levels of $\mathrm{NO}_{x}$ in $\mathrm{SS}$ and AA individuals were $10 \pm 8 \mu \mathrm{M}$ and $12 \pm 15 \mu \mathrm{M}$, respectively. After 2 hours of inhalation (hours 3 and 4; filled horizontal bar), mean levels rose significantly $(P<0.001)$ to $85 \pm 8 \mu \mathrm{M}$ in the SS individuals and 60 $\pm 15 \mu \mathrm{M}$ in the AA individuals. After NO inhalation, there was a steady decrease in plasma $\mathrm{NO}_{x}$ concentration. (b) During $\mathrm{NO}$ inhalation (hours 3 and 4; filled horizontal bar), mean methemoglobin levels rose from $0.3 \pm 0.0 \%$ in the SS individuals and $0.24 \pm 0.02 \%$ in the AA individuals to $1.2 \pm 0.4 \%$ and $0.9 \pm 0.09 \%$, respectively $(P=0.001)$. After correcting for hemoglobin concentration, there was no difference in absolute methemoglobin values between AA and SS individuals. Values returned to baseline within 1-2 hours in all individuals.

on an HP1100 integrated LC-electrospray system (Hewlett-Packard, Palo Alto, California, USA) using a $2.1 \mathrm{~mm} \times 15 \mathrm{~cm} \mathrm{C} 3$ Zorbax reverse-phase chromatography column at $20^{\circ} \mathrm{C}$ and a flow rate of $0.3 \mathrm{~mL} / \mathrm{min}$. Solvent $A$ was $5 \%$ acetic acid in water, and solvent $B$ was acetonitrile. Separation of the globin proteins (minus the heme groups) was achieved using a linear gradient of $33-37 \%$ solvent B over 25 minutes. The mass spectrum was scanned at $500-1,700 \mathrm{~m} / z$ at $0.1-\mathrm{m} / z$ increments every 2 seconds while acquiring full-profile data with the fragmentor at $30 \mathrm{~V}$. To determine which cysteine binds $\mathrm{NO}$ on the globin chains, each sample was digested with endoproteinase Glu-C (Boehringer Mannheim Biochemicals, Indianapolis, Indiana, USA) in ammonium acetate ( $\mathrm{pH} 4.0)$ at a hemoglobin/enzyme ratio of $10: 1$, in the presence of $5 \%$ acetonitrile (to denature proteins), for 2 hours at $25^{\circ} \mathrm{C}$. Samples were then run on a C3 column. The solvent was held isocratic ( $2 \%$ acetonitrile, $0.3 \mathrm{~mL} / \mathrm{min}$ ) for 5 minutes and then ramped to 
Table 1

Baseline characteristics

\begin{tabular}{|c|c|c|}
\hline & Sickle cell & Normal \\
\hline \multicolumn{3}{|l|}{ Race, $n(\%)$} \\
\hline African American & $7(100 \%)$ & $3(60 \%)$ \\
\hline Caucasian & & $2(40 \%)$ \\
\hline \multicolumn{3}{|l|}{ Gender, $n(\%)$} \\
\hline Male & $3(43 \%)$ & $3(60 \%)$ \\
\hline Female & $4(57 \%)$ & $2(40 \%)$ \\
\hline Age (years), median (min, max) & $36(21,40)$ & $32(25,45)$ \\
\hline Rate of general pain crises (per month), median (min, max) & $4.0(0.3,5.0)$ & Not applicable \\
\hline Rate of emergency department pain crises (per month) ${ }^{\mathrm{A}}$, median (min, max) & $0.3(0.0,1.0)$ & Not applicable \\
\hline Hemoglobin S-only phenotype ${ }^{B}$ & $7 / 7$ & \\
\hline Fetal hemoglobin (\%), median ( $\min , \max )$ & $7.6(2.0,32.5)$ & Not measured \\
\hline Hemoglobin A2 (\%) & 4.4 & Not measured \\
\hline Hemoglobin (g/dL), median (min, max) & $8.3(6.8,9.0)$ & $14.0(12.3,15.5$ \\
\hline Hematocrit (\%), median (min, $\max )$ & $24.5(19.4,27.0)$ & $41.2(38.1,45.7$ \\
\hline Reticulocytes (\%), median (min, max) & $11.0(6.2,30.8)$ & Not measured \\
\hline Mean corpuscular volume $\left(\mu \mathrm{m}^{3}\right)$, median ( $\left.\min , \max \right)$ & $91.6(76.0,106.0)$ & $86.0(84.0,91.0$ \\
\hline P50 (deoxygenation) $)^{\mathrm{D}}(\mathrm{mmHg})$ median $(\mathrm{min}, \mathrm{max})$ & $32.2(25.2,34.5)$ & $27.1(26.0,27.9$ \\
\hline P50 (oxygenation) $)^{\mathrm{E}}(\mathrm{mmHg})$ median $(\min , \max )$ & $36.0(29.5,38.4)$ & $27.2(26.5,28.2$ \\
\hline White blood cells count $\left(\mathrm{K} / \mu \mathrm{m}^{3}\right)$, median $(\min , \max )$ & $9.8(7.0,15.2)$ & $4.7(3.9,6.1)$ \\
\hline Platelet count $\left(\mathrm{K} / \mu \mathrm{m}^{3}\right)$, median $(\min , \max )$ & $459(233,584)$ & $211(180,225)$ \\
\hline
\end{tabular}

${ }^{A}$ Defined as a pain crisis requiring a visit to the emergency department or hospital. BTwo patients may have HbS- $\beta^{0}$ thalassemia, based on low mean corpuscular volume and an increased percentage of hemoglobin $\mathrm{F}$ and/or A2. ${ }^{\mathrm{C}}$ Measured on only 1 patient. ${ }^{\mathrm{D}} \mathrm{P}_{50}$ measured during the deoxygenation of blood sample (see Methods). ${ }^{E} \mathrm{P}_{50}$ measured during the oxygenation of blood sample (see Methods).

$18 \%$ acetonitrile over 15 minutes. The mass spectrometer was scanned at $200-1,700 \mathrm{~m} / z$ every 4 seconds. The fragment nitrosylated at $\beta$-chain cysteine 93 was identified as a mass of $1,334.5$. This was 29 mass units higher than $1,305.5$, the molecular weight of the protein fragment containing $\beta$-chain amino acids 91-101.

Chemiluminescent detection of SNO-hemoglobin. The method of Zweier and colleagues for the measurement of nitrite and $S$-nitrosothiols by reaction with $\mathrm{I}_{3}{ }^{-}$to release $\mathrm{NO}$ gas was used (12). Briefly, $7 \mathrm{~mL}$ of glacial acetic acid and $2 \mathrm{~mL}$ of distilled water are mixed with 50 $\mathrm{mg}$ of $\mathrm{KI}$ (or $\mathrm{NaI}$ ). A crystal of $\mathrm{I}_{2}$ is added to yield a concentration of 6-20 mM. Helium is bubbled through the reaction mixture, through $1 \mathrm{~N} \mathrm{NaOH}$, and then into the Sievers Model $280 \mathrm{NO}$ analyzer. Standard curves were obtained using $S$-nitrosoglutathione (Sigma Chemical Co., St. Louis, Missouri, USA) and SNO-hemoglobin (synthesized as outlined later here). The chemiluminescent signal from the $\mathrm{I}_{3}{ }^{-}$reaction to form $\mathrm{NO}$ is linear at greater than 1.0 pmol (data not shown) with both nitrite ions $\left(r^{2}=0.9997, P<0.001\right)$ and several $S$-nitrosothiols ( $S$-nitrosoglutathione, $S$-nitrosocysteine, and $S$-nitroso- $N$-acetylcysteine; average $r^{2}=0.9989, P<$ 0.001). Nitrate ions do not react.

Standardization of measurement of SNO-hemoglobin by $\mathrm{I}_{3}{ }^{-}$release of $\mathrm{NO}$ was made by comparison of synthesized SNO-hemoglobin, by reaction of hemoglobin with $S$-nitrosocysteine as described by McMahon and Stamler (13), with mass spectrometry measurements. Synthesized SNO-hemoglobin had a mean $1.96 \pm 0.17$ moles of $\mathrm{NO}$ per hemoglobin tetramer $(n=$ 15), consistent with nitrosylation of a single reactive thiol (cysteine 93) on the two $\beta$-chains of hemoglobin. There was significant correlation between the methods for quantification of SNO-hemoglobin $(r=0.63, n=14$, $P<0.05)$; however, measurements of SNO-hemoglobin by mass spectrometry consistently underestimated the level of nitrosylation. To determine the limits of sensitivity of the chemiluminescence assay, the SNO-hemoglobin was serially diluted in oxyhemoglobin $(1 \mathrm{mM})$ from $3 \%$ to $0.001 \%$ SNO-hemoglobin/oxyhemoglobin (concentration in terms of heme subunit). Release of NO was linear over this range $\left(r^{2}=0.996, P<0.001\right)$. Similar results were obtained from several preparations of hemoglobin S.

$\mathrm{I}_{3}{ }^{-}$release of $\mathrm{NO}$ from the heme in nitrosyl(heme)hemoglobin was measured after forming the latter by reacting $\mathrm{NO}$ gas with deoxyhemoglobin. Nitrosyl(heme)hemoglobin refers to hemoglobin with an $\mathrm{NO}$ bound to at least 1 of the heme groups (the heme, in parenthesis, is included for clarification). Briefly, purified oxyhemoglobin was deoxygenated in nitrogen for 2 hours, followed by a 45 -minute exposure to $100 \% \mathrm{NO}$ gas (bubbled through $5 \mathrm{~N} \mathrm{NaOH}$ to remove $\mathrm{NO}_{X}$ species, i.e., nitrate/nitrite). Formation of nitrosyl(heme)hemoglobin was confirmed by full-scan optical spectroscopy, demonstrating a Soret peak at $417 \mathrm{~nm}$, the $\beta$ band at $544 \mathrm{~nm}$, the $\alpha$ band at $572 \mathrm{~nm}$, and increased absorption (compared with oxyhemoglobin) at $560 \mathrm{~nm}$. Mass spectrometry measurements confirmed that NO gas under these conditions did not nitrosate the globin chains. It was observed that the $\mathrm{I}_{3}{ }^{-}$ chemiluminescence assay detects NO bound to heme (90\% yield; $n=3$ ). 
During in vivo experiments, patient samples, after Sephadex G25 processing to remove nitrite contamination, were injected in duplicate $(200 \mu \mathrm{L}$ sample volume of approximately $1 \mathrm{mM}$ hemoglobin) into excess $\mathrm{I}_{3}{ }^{-}$in the purge vessel. The residual signal represented release of NO from the S-NO bond of SNO-hemoglobin, with possible contribution from the nitrosyl(heme)hemoglobin. The concentration of NO released in $\mathrm{nM}$ is subtracted from the NO concentration generated by $200 \mu \mathrm{L}$ injection of the water from the Sephadex G25 column (background control), obtained immediately before running the hemoglobin sample on the column. This value is divided by the concentration of heme subunit, and the total is multiplied by 100 .

Standard laboratory tests. Arterial and venous blood gas analysis (278 Blood Gas System; Ciba-Corning Diagnostics Corp., Medfield, Massachusetts, USA) and cooximetry (270 Co-Oximeter; Ciba-Corning) were immediately performed (<5-minute delay) after obtaining venous and arterial samples from the volunteers to determine $\mathrm{pH}, \mathrm{PO}_{2}, \mathrm{PCO}_{2}$, hemoglobin saturation, and carboxy- and methemoglobin concentrations. Methemoglobin concentrations using the Co-Oximeter were validated by comparison with measurements by absorption spectroscopy at 700, 630, 576, and $560 \mathrm{~nm}$ using the Winterbourn equation (14).

Statistical analysis. The Wilcoxon test was used for experiments comparing AA and SS individuals breathing 80 ppm NO for 2 hours. Within each group (AA or SS), a signed-rank test determined significance of observed changes. For dose-titration experiments of inhaled NO, because of the high precision of the measurements, a 2-tailed paired $t$ test was used to analyze increases in the mean values of paired samples before and after NO inhalation, as well as to analyze the significance of differences in nitrosylated hemoglobin levels between arterial and venous blood at baseline and on dose titration of inhaled NO.

\section{Results}

Baseline characteristics of the normal volunteers and the individuals with sickle cells. Baseline characteristics of the normal volunteers and the individuals with sickle cells are outlined in Table 1 . The 7 individuals with sickle cell anemia were all of hemoglobin S-only phenotype, with hemoglobin F levels in the range of $2-32.5 \%$. Two of the individuals may have $\mathrm{HbS}-\beta^{0}$ thalassemia or sickle cell anemia- $\alpha$ thalassemia.

Clinical and laboratory parameters. There was no observed effect of NO inhalation on vital signs, electrocardiogram, arterial or venous blood $\mathrm{pH}, \mathrm{PaO}_{2}$, $\mathrm{PaCO}_{2}$, or oxygen saturation (data not shown). Before $\mathrm{NO}$ inhalation, the SS arterial $\mathrm{pO}_{2}$ was lower than the AA arterial $\mathrm{pO}_{2}$. The $\mathrm{SS}$ venous $\mathrm{pO}_{2}$ values are similar to those of AA individuals. This narrow difference between arterial and venous $\mathrm{pO}_{2}$ levels in SS patients is indicative of the reduced oxygen extraction or arterialization of venous blood that is characteristic of patients with sickle cell anemia. Inhaled NO did not improve the baseline arterial and venous hypoxemia that was observed in SS individuals, compared with AA individuals, when administered as 80 ppm for 2 hours (Figure 1a) or in increasing concentrations of 40, 60, and 80 ppm given each for 1 hour (Figure 1b). The $\mathrm{FiO}_{2}$ was maintained at 0.21 with an oxygen blender when delivering $80 \mathrm{ppm}$ NO. There were no significant changes observed in hemoglobin concentration, hematocrit, platelet count, white blood cell count, or mean corpuscular volume after NO delivery at 80 ppm for 2 hours (data not shown).

NO metabolism. Inhaled $\mathrm{NO}$ at $80 \mathrm{ppm}$ resulted in a substantial increase in $\mathrm{NO}_{\mathrm{X}}$, which was predominantly nitrate. Mean baseline plasma levels of $\mathrm{NO}_{\mathrm{X}}$ in SS and AA individuals were $10 \pm 8 \mu \mathrm{M}$ and $12 \pm 15 \mu \mathrm{M}$, respectively. At 1 and 2 hours of inhalation, mean $\mathrm{NO}_{\mathrm{X}}$ levels significantly rose to $85 \pm 8 \mu \mathrm{M}$ in the SS indi-

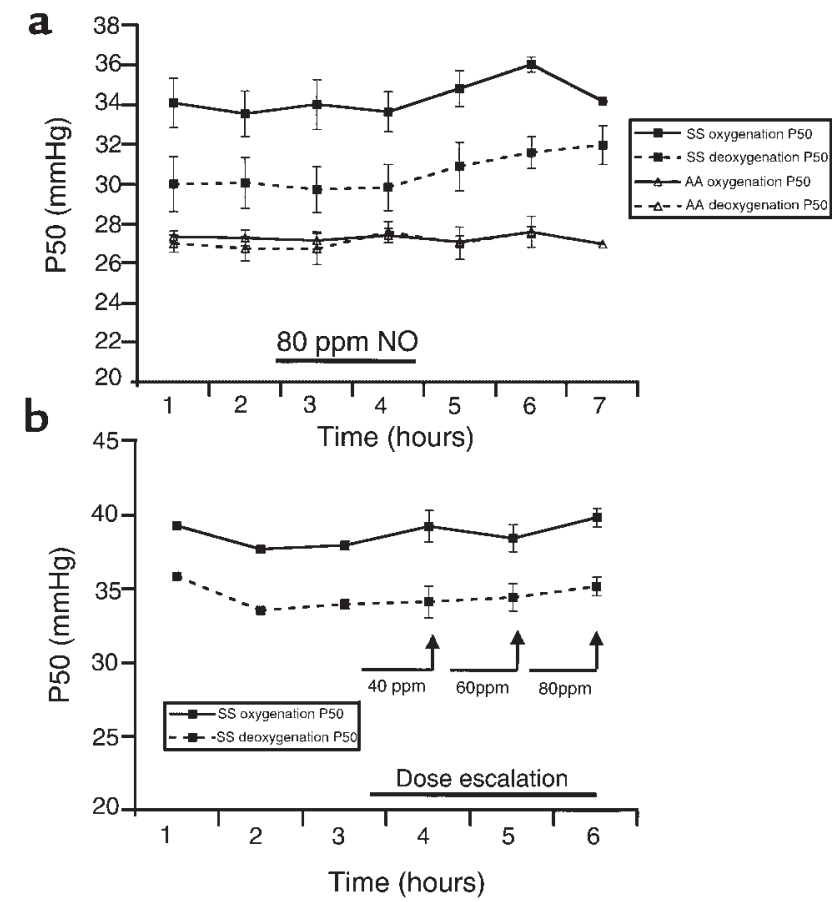

Figure 3

Effect of inhaled NO on the oxygen affinity $\left(P_{50}\right)$ of SS and AA erythrocytes. (a) The AA ODCs (expressed as $\mathrm{P}_{50}$ ) at baseline before NO inhalation (hours 1 and 2) demonstrated minimal hysteresis when measured during sample deoxygenation or during sample oxygenation. The baseline SS ODCs measured at 1 and 2 hours before NO inhalation, as expected, demonstrated significant hysteresis during sample deoxygenation and during sample oxygenation. There was no significant change in any of the $\mathrm{P}_{50}$ measurements after 1 and 2 hours of inhaled NO at $80 \mathrm{ppm}$ in either the AA or SS individuals (hours 3 and 4; filled horizontal bar), measured either during sample deoxygenation (for SS individuals; $P=0.63$ ) or reoxygenation (for SS individuals; $P=1.00$ ). There was no significant change in any of the $P_{50}$ measurements for up to 3 hours after $\mathrm{NO}$ inhalation in any of the individuals tested (10/10 tested in the first hour after $\mathrm{NO} ; 7 / 10$ in the second hour; and 3/10 in the third hour). (b) During dose escalation of inhaled NO in 3 SS individuals, there is no significant change in $\mathrm{P}_{50}$ measured during sample deoxygenation or reoxygenation after 1 hour inhalation each of 40,60, and 80 ppm NO gas. 

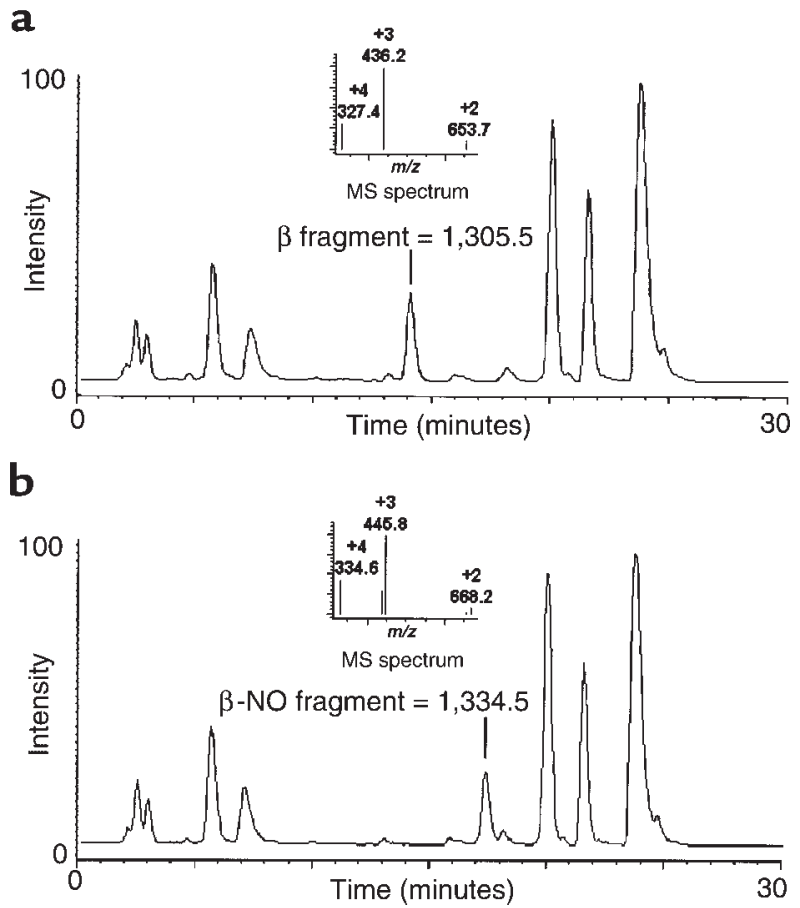

Figure 4

HPLC-electrospray ionization mass spectrometry of SNO-hemoglobin digested with endoproteinase Glu-C confirms specificity of Snitrosocysteine reaction with hemoglobin $\beta$-chain cysteine 93 . (a) Shown is the characteristic chromatogram, after enzymatic digestion of hemoglobin, of the 11-amino acid fragment (hemoglobin $\beta$-chain amino acids 91-101) that includes cysteine 93. The molecular weight is $1,305.5$, with 3 predominant charge states $(+4,+3,+2)$ with respective $\mathrm{m} / \mathrm{z}$ of $327.4,436.2$, and 653.7 (inset). (b) Digestion of SNO-hemoglobin results in an increased retention time on the column and an increase in the molecular weight of the 11-amino acid fragment to $1,334.5$, consistent with the addition of 29 mass units (NO has 30 mass units minus 1 mass unit of hydrogen lost during covalent binding). The $m / z$ values of the 3 charge states $(+4,+3,+2)$ increase to $334.6,445.8$, and 668.2, respectively (inset).

viduals and $60 \pm 15 \mu \mathrm{M}$ in the $\mathrm{AA}$ individuals $(P<$ 0.001) (Figure 2a). Mean methemoglobin levels, obtained by the Co-Oximeter, rose from $0.3 \pm 0.0$ in the SS individuals and $0.24 \pm 0.02 \%$ in the AA individuals to $1.2 \pm 0.4 \%$ and $0.9 \pm 0.09 \%$, respectively $(P=$ 0.001) (Figure 2b).

ODC measurements. The AA ODCs at baseline before $\mathrm{NO}$ inhalation demonstrated minimal hysteresis when measured during deoxygenation $\left(\mathrm{P}_{50}\right.$ measured before inhaled $\mathrm{NO}=27.0 \pm 0.4 \mathrm{mmHg}$ ) or during oxygenation $\left(\mathrm{P}_{50}\right.$ measured before inhaled $\left.\mathrm{NO}=27.3 \pm 0.3 \mathrm{mmHg}\right)$. As expected, the baseline SS ODCs measured before NO inhalation demonstrated significant hysteresis, with $\mathrm{P}_{50}$ measurements during deoxygenation of $30 \pm$ $1.4 \mathrm{mmHg}$ and during oxygenation of $34.1 \pm 1.3$ $\mathrm{mmHg}$. There was no significant change in any of the $\mathrm{P}_{50}$ measurements at 1 and 2 hours of $80 \mathrm{ppm}$ inhaled $\mathrm{NO}$ in either the AA or SS individuals, measured either during deoxygenation (for SS individuals; $P=0.63$ ) or reoxygenation (for $\mathrm{SS}$ individuals; $P=1.00$ ). There was no significant change in any of the $\mathrm{P}_{50}$ measurements for up to 3 hours after NO inhalation in any of the individuals tested (Figure $3 \mathrm{a}$ ). In 3 further studies ( 2 new patients and 1 previously studied) of 40,60 , and 80 ppm NO dose escalation, there was no change in the $\mathrm{P}_{50}$ (Figure $3 \mathrm{~b}$ ). No changes in ODC characteristics or partial pressure of oxygen at which $10 \%$ of the hemoglobin is saturated with oxygen $\left(\mathrm{P}_{10}\right)$ were observed. There was no correlation between absolute methemoglobin formation and measured $\mathrm{P}_{50}(r=0.2, P=0.80)$.

Measurement of modification of the $\beta$-chain cysteine 93 by HPLC-electrospray mass spectrometry. Electrospray mass spectrometry of synthesized SNO-hemoglobin demonstrated a single NO adduct on the $\beta$-globin protein chains at levels in the range of $15-30 \%$. Digestion with endoproteinase Glu-C confirms specificity of cysteineNO reaction with hemoglobin $\beta$-chain cysteine 93 . Figure $4 \mathrm{a}$ demonstrates the characteristic chromatogram, after enzymatic digestion of hemoglobin, of the 11 -amino acid fragment (hemoglobin $\beta$-chain amino acids 91-101) that includes cysteine 93 . The NO-modified fragment (Figure $4 \mathrm{~b}$ ) demonstrates 3 prominent charge states $(+2,+3,+4)$ and an increased retention time on the column. Reaction with the remaining thiol ( 2 total) on the $\beta$ chain and the single thiol on the $\alpha$ chain does not occur, unless the hemoglobin is denatured (reactions at low $\mathrm{pH}$ ). On study days, patient samples were analyzed immediately upon collection using full-scan measurements of the $\beta$ chain and proteolytic digestion with analysis of modification of the 91-101 amino acid fragment. No adducts larger than 29 mass units (consistent with glutathione or cysteine) were observed before, during, or after $\mathrm{NO}$ inhalation. The technique has limited sensitivity (to $1-2 \%$ modification by NO) owing to interference by the 22 -mass unit sodium. Proteolytic digestion increases the signal/noise ratio, but loss of NO occurs before complete digestion, again limiting sensitivity. These results, however, are inconsistent with most of the mechanisms postulated to explain the reported increase in oxygen affinity with NO exposure (9).

Measurement of nitrosylation of hemoglobin by chemiluminescent $\mathrm{I}_{3}{ }^{-}$reaction. At baseline, the arterial and venous nitrosylated hemoglobin levels in the SS individuals were not significantly different. For the 3 hours before NO inhalation, the mean percentage of nitrosylated hemoglobin in the arterial blood was about $0.004 \%$, and in the venous blood, about $0.004 \%$. There is a dose-dependent increase in nitrosylated hemoglobin (expressed as percent moles of NO per moles of heme subunit) during $\mathrm{NO}$ inhalation in both arterial blood $(0.011 \%$ on $40 \mathrm{ppm}, 0.016 \%$ on $60 \mathrm{ppm}$, and $0.022 \%$ on $80 \mathrm{ppm})$ and venous blood $(0.014 \%$ on $40 \mathrm{ppm}, 0.016 \%$ on $60 \mathrm{ppm}$, and $0.016 \%$ on $80 \mathrm{ppm}$ ). A significant arterial-venous difference was observed on $80 \mathrm{ppm}$ inhaled NO $(P=0.02)$. The results of experiments from 1 individual, as a representative example, are shown in Figure 5, a-d, and the mean values for all 3 SS individuals are shown in Figure 6. 


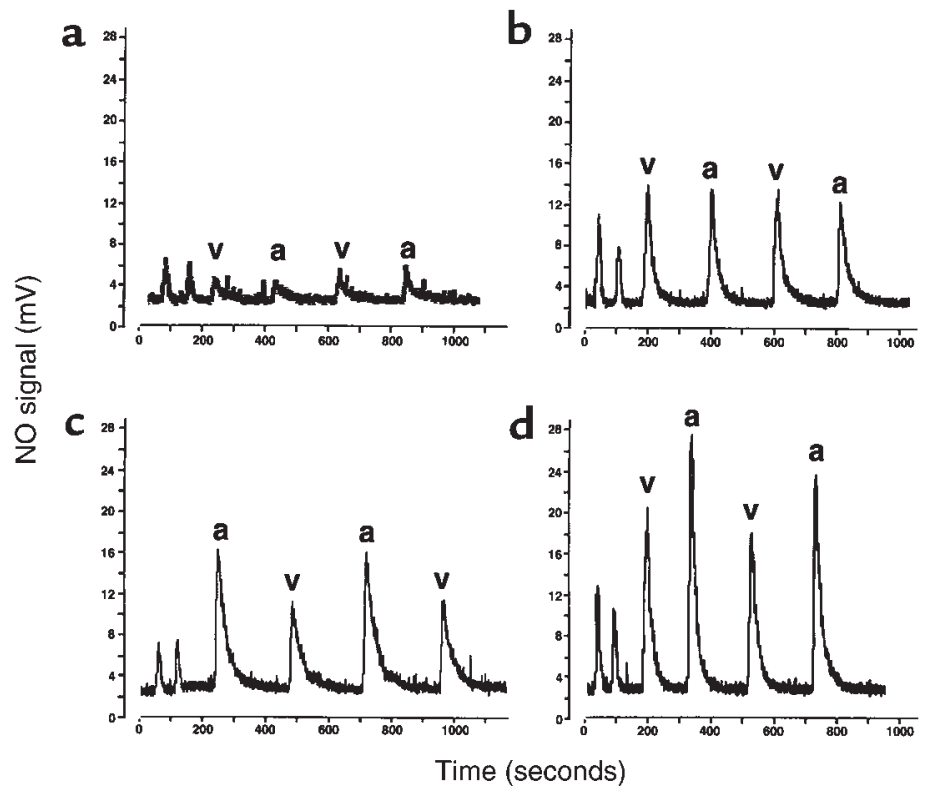

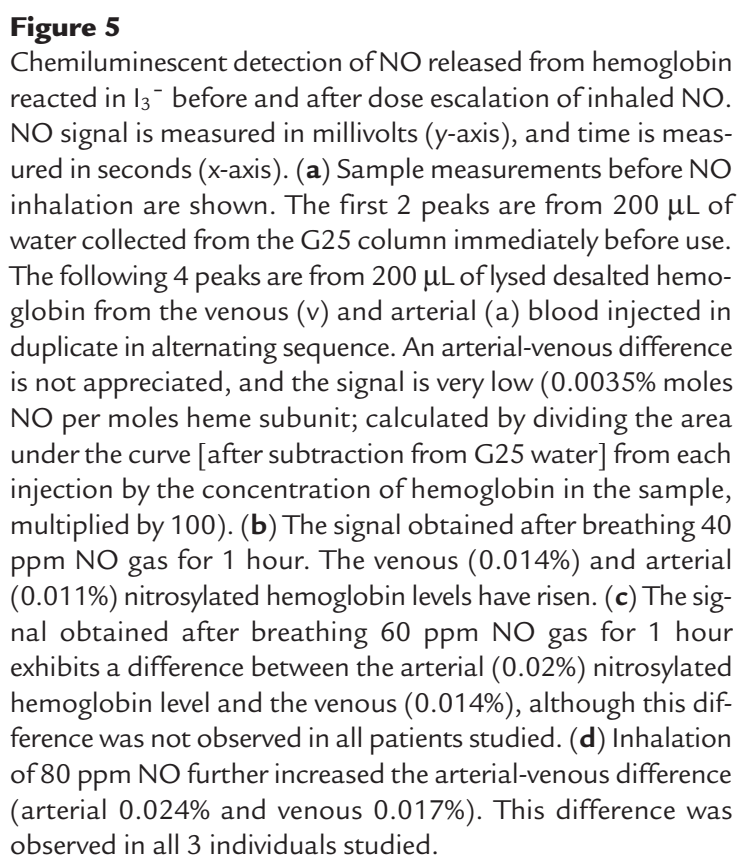

Therefore, inhaled NO was well tolerated and produced no changes in hemodynamics or oxygenation. NO inhalation resulted in a significant reaction with the ferrous heme of oxyhemoglobin to produce nitrate and methemoglobin and, to a lesser extent, nitrosylated the hemoglobin molecule. The specificity of the NO donor $S$-nitrosocysteine for reaction with the hemoglobin $\beta$-chain cysteine 93 observed in vitro using mass spectrometry suggests that the observed increase in nitrosylated hemoglobin represents SNO-hemoglobin but could also represent nitrosyl(heme)hemoglobin. The levels of nitrosylated hemoglobin observed during NO inhalation were low, and there was no increase in oxygen affinity (measured by a reduction in $\mathrm{P}_{50}$ ). No modification of hemoglobin with glutathione was observed during NO breathing.

\section{Discussion}

This study was designed to test recent reports that inhaled NO increases the oxygen affinity of sickle cell erythrocytes, and to test whether inhaled NO reacts with the $\beta$-chain cysteine 93 to form SNO-hemoglobin. Our data suggest that in both normal (AA) and sickle cell (SS) individuals, up to 2 hours of inhaled NO at 80 ppm is well tolerated, with minimal acute cardiovascular or hematological effects, and can be monitored by increased formation of plasma $\mathrm{NO}_{x}$ compounds and small increases in methemoglobin. The metabolic fate of inhaled NO appears to be similar in SS and AA individuals, although we did observe a nonsignificant trend toward higher level $\mathrm{NO}_{\mathrm{x}}$ production in the SS individuals. We were unable to detect any change in hemoglobin oxygen affinity in either the normal patients or those with sickle cell; nor did we find any effect on arterial or venous $\mathrm{pO}_{2}$ in these individuals. We did demonstrate, however, increased levels of NO bound to hemo- globin in both arterial and venous blood from the subjects with sickle cell, and believe that this may represent the $S$-nitrosated cysteine 93 residue of the hemoglobin $S \beta$ chain.

The lack of an observed increase in oxygen affinity ( $\mathrm{P}_{50}$ reduction) is consistent with our observation that inhaled NO produces only modest increases in methemoglobin and nitrosylated hemoglobin. It has been known for more than half a century that methemoglobin in high levels produces an apparent increase in the oxygen affinity of hemoglobin, but this effect is negligible at the levels of methemoglobin observed in this study. Because of the proposed allosteric interaction of oxygen and NO on hemoglobin, SNO-hemoglobin should exhibit an increase in oxygen affinity independent of effects on polymerization. Indeed, a recent report described an increased oxygen affinity of AA SNO-hemoglobin directly proportional to the percentage of modified $\beta$-chain cysteine 93 (15). However, these data suggest that a large fraction of the hemoglobin molecules would require modification to significantly change hemoglobin oxygen affinity, and we observed only a mean $0.017 \%$ increase in the arterial hemoglobin that was nitrosylated. In previous reports, the formation of a mixed disulfide, such as glutathionyl-hemoglobin, at the highly conserved 93 cysteine on the $\beta$ chain, interfered with polymerization, increased oxygen affinity, and inhibited sickling. However, this effect required a high proportion of reacted hemoglobin (in the range of $8-25 \%$ labile glutathionylhemoglobin and more than $95 \%$ irreversible covalent modification with thiols) $(16,17)$. It remains a possibility that inhaled NO catalyzes the formation of mixed disulfides of glutathione or other molecules, with subsequent sulfhydryl-mixed disulfide exchange to the $\beta$ chain cysteine 93. However, using HPLC-mass spec- 


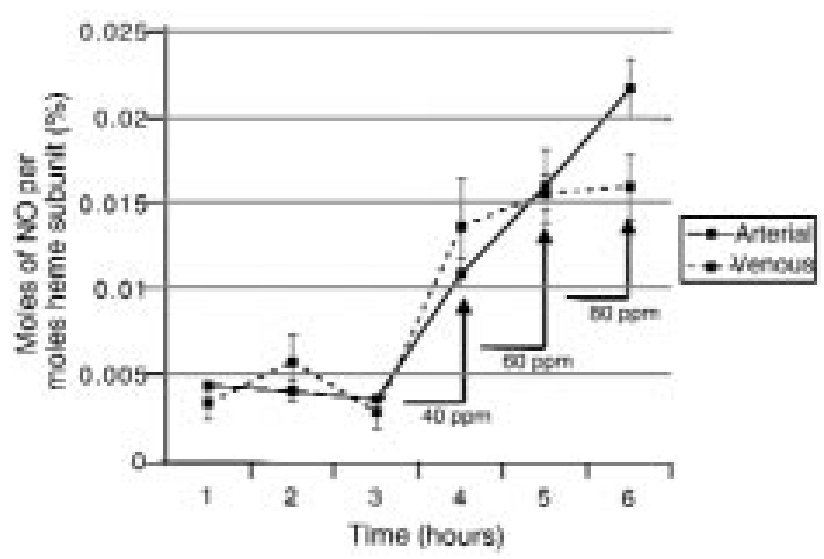

\section{Figure 6}

Effect of dose escalation of inhaled NO on nitrosylated hemoglobin, expressed as the percentage moles of NO per moles of heme subunits. For 3 hours before NO inhalation, the mean percentage of nitrosylated hemoglobin in the arterial blood was $0.004 \%$ and in the venous blood, $0.004 \%$. There is a significant stepwise increase in nitrosylated hemoglobin during $\mathrm{NO}$ inhalation in both arterial ( $0.011 \%$ on 40 ppm, $0.016 \%$ on 60 ppm, and $0.022 \%$ on 80 ppm) and venous $(0.014 \%$ on $40 \mathrm{ppm}, 0.016 \%$ on $60 \mathrm{ppm}$, and $0.016 \%$ on $80 \mathrm{ppm}$ ) blood ( $P<0.05$ for all increases except the venous sample at $40 \mathrm{ppm}$, for which $P=0.075)$. A significant arterial-venous difference was observed on $80 \mathrm{ppm}$ inhaled $\mathrm{NO}(P=0.02)$.

troscopy, we did not observe any molecular weight adducts consistent with glutathionyl-hemoglobin or any other mixed disulfide. Thus, other effects of NO adducts on oxygen affinity - directly, by changing the $\mathrm{T} \rightarrow \mathrm{R}$ conformational changes in hemoglobin; or indirectly (in the case of hemoglobin $S$ ), by interfering with subunit contacts in the polymer phase - are unlikely at the detected levels of nitrosylation.

There is no apparent explanation for the discrepancy between the results presented in this article and those of Head et al. (9). We calculated $95 \%$ confidence intervals, using $t$ distributions (assuming normality), for the mean change in $\mathrm{P}_{50}$ presented here (for the first series of experiments, $n=5$ ) and for that of Head et al., and found similar results for the AA individuals. However, there was no overlap in the confidence intervals between the SS individuals presented here $(-0.86,0.91)$ and those presented by Head et al. $(-6.23,-2.97)$. This suggests that this discrepancy cannot be explained by patient sampling variability. In parallel experiments, we have found that treating AA or SS erythrocytes with $\mathrm{NO}$ gas and NO donor compounds only changes oxygen affinity at levels in which high levels of methemoglobin or SNO-hemoglobin are formed (B.W. Hrinczenko, A. Alayash, M.T. Gladwin, and A.N. Schechter, unpublished study). Although it is expected that higher levels of modified cysteine 93 are needed to inhibit hemoglobin S polymerization and improve oxygen affinity, it is possible that small quantities of SNOhemoglobin would have significant peripheral physiological effects. NO, carried as SNO-hemoglobin to the microvasculature, may modulate vascular tone (6), inhibit platelet activation (18), and inhibit leukocyte Pselectin-dependent adhesion and migration (19). These actions could potentially have a favorable impact on the pathophysiology of sickle cell anemia, a disease characterized by major abnormalities in microvascular perfusion; hypercoagulability; and increased expression of erythrocyte, endothelial, and leukocyte adhesion molecules (20). Anecdotally, inhaled NO has been described to improve oxygenation and pulmonary hypertension and to shorten the course of the acute chest syndrome (21). Sickle cell anemia animal models further support the role of $\mathrm{NO}$ in improving cerebral vascular perfusion (22).

Recent work by Stamler and colleagues (6-8) suggests that NO binding to hemoglobin may play a role in the regulation of vascular tone, with NO binding and release tied to oxygen-induced allosteric structural transitions. In the lungs, hemoglobin is highly saturated with oxygen, and NO produced in the lungs is thought to bind to cysteine 93 on the $\beta$ chain. This SNO-hemoglobin is carried by the red cells to the microvascular system, where oxygen tensions are reduced. After deoxygenation, allosteric structural changes in the hemoglobin molecule favor the release of NO, which diffuses to the arterial wall, possibly via thiol intermediaries such as glutathione, and causes vasodilation. If this model is correct, it would suggest that as a result of a direct vasodilatory effect of $\mathrm{NO}$ in the periphery, therapeutic delivery of $\mathrm{NO}$ in the form of SNO-hemoglobin may be beneficial to patients with sickle cell anemia who have impaired microvascular perfusion.

To date, this model is supported by observations in vitro using rat and human hemoglobin and whole erythrocytes (6). In vivo, increased erythrocyte SNO-hemoglobin in arterial blood compared with venous blood has been demonstrated in Sprague-Dawley rats (6), and recent studies have demonstrated an increase in SNOhemoglobin in oxygenated blood (venous) compared with deoxygenated blood (arterial) in the human fetalplacental circulation (venous blood carries oxyhemoglobin from placenta to fetus in the fetal circulation) (23). Our observation of increased nitrosylation of human hemoglobin S upon inhalation of $\mathrm{NO}$, and the formation of a significant arterial-venous difference in response to such inhalation, provides new evidence that hemoglobin stabilizes and transports NO. The specificity of the in vitro reaction of cysteine-NO with $\beta$ chain cysteine 93 , evidenced by mass spectrometry experiments (Figure 4), argues that the observed in vivo nitrosylated hemoglobin represents SNO-hemoglobin. These data support the theory that human cysteine 93 participates in a covalent interaction with $\mathrm{NO}$ in the human lung, and that therapeutic inhalation of $\mathrm{NO}$ can augment $S$-nitrosation at this site.

It remains possible that the levels of nitrosylated hemoglobin measured before and after NO inhalation are attributable to the measurement of $\mathrm{NO}$ release from heme rather then from the cysteine 93 residue. However, resting measurements of nitrosyl(heme)hemoglobin by 
Stamler and colleagues document that basal nitrosyl(heme)hemoglobin content is actually higher in venous than arterial blood in the Sprague-Dawley rat (6). Takahashi et al. (24) performed electron paramagnetic (EPR) measurements of nitrosyl(heme)hemoglobin content of arterial and venous blood from sheep breathing $60 \mathrm{ppm}$ $\mathrm{NO}$ and found no significant arterial-venous differences. The nitrosyl(heme)hemoglobin content of these sheep constituted less than $0.11 \%$ modification of total hemoglobin. This value is 10 -fold higher than the signal measured in this current study, and it is therefore possible that EPR would be incapable of detecting the arterial-venous difference of $0.005 \%$ that we have measured. Although the weight of evidence suggests specificity for cysteine 93 $S$-nitrosation, it remains possible that part of our measured arterial-venous difference in nitrosylated hemoglobin is attributable to nitrosyl(heme)hemoglobin rather than SNO-hemoglobin.

It is not clear why there is no observed baseline arterial-venous difference of nitrosylated hemoglobin in SS erythrocytes, as has been previously suggested for AA erythrocytes, or why this difference widens only after inhalation of relatively high levels of NO. This may be the result of a small sample size and difficulty in measuring such low levels of bound NO. With further studies, a baseline arterial-venous difference may become apparent. Alternatively, the narrow arterial-venous difference may be unique to sickle cell disease because of the classically described reduced arterial-venous oxygen difference, which further narrows during crisis $(25,26)$. Note that in Figure 1a, the individuals with sickle cell have a reduced arterial $\mathrm{pO}_{2}$, whereas venous $\mathrm{pO}_{2}$ is similar to or higher than the AA values. This phenomenon is attributable to the arterialization of venous blood due to regional obstruction of flow causing shunting into larger caliber arterial-venous anastomoses. A second mechanism may be a reduced oxygen extraction secondary to an increased cardiac output from anemia or altered microvascular perfusion. It is interesting to speculate that this reduced arterial-venous oxygen difference would similarly affect the SNO-hemoglobin difference because of oxygen-NO allostery.

In conclusion, inhaled NO reacts with hemoglobin in SS and AA individuals, resulting in significant increases in $\mathrm{NO}_{\mathrm{x}}$ and mild elevations in methemoglobin. We observed no change in hemoglobin oxygen affinity or arterial and venous $\mathrm{pO}_{2}$ levels. Importantly, we report nitrosylation of hemoglobin $\mathrm{S}$, possibly representing $\beta$-chain SNO-hemoglobin, during NO breathing - supporting an in vivo role for the human hemoglobin $S, \beta$ chain cysteine 93 that includes covalent binding of NO. The level of nitrosylation appears to be too low to affect overall sickle cell oxygen affinity, but may provide a mechanism to augment NO delivery to the microvasculature and possibly improve microvascular perfusion.

\section{Acknowledgements}

We thank our patients with sickle cell anemia for their support and enthusiasm for this project, and A. Suffre- dini and R. Danner, who helped conceive and execute important components of this study. We also acknowledge L. Keefer, D. Wink, and J. Nawrocki for providing their expertise with NO chemistry; the Critical Care Medicine Department respiratory therapy section for assistance with NO delivery and blood gas analysis; and E. Link for helping with patient recruitment and community outreach efforts.

1. Bunn, H.F. 1997. Pathogenesis and treatment of sickle cell disease. $N$. Engl. J. Med. 337:762-769.

2. Steinberg, M.H. 1999. Management of sickle cell disease. N. Engl.J. Med. 340:1021-1030.

3. Gaston, B., et al. 1993. Endogenous nitrogen oxides and bronchodilator Snitrosothiols in human airways. Proc. Natl. Acad. Sci. USA. 90:10957-10961.

4. Stamler, J.S., et al. 1992. Nitric oxide circulates in mammalian plasma primarily as an S-nitroso adduct of serum albumin. Proc. Natl. Acad. Sci. USA. 89:7674-7677.

5. Arnelle, D.R., and Stamler, J.S. 1995. NO+, NO, and NO- donation by Snitrosothiols: implications for regulation of physiological functions by S-nitrosylation and acceleration of disulfide formation. Arch. Biochem. Biophys. 318:279-285.

6. Jia, L., Bonaventura, C., Bonaventura, J., and Stamler, J.S. 1996. S-nitrosohaemoglobin: a dynamic activity of blood involved in vascular control. Nature. 380:221-226.

7. Gow, A.J., and Stamler, J.S. 1998. Reactions between nitric oxide and haemoglobin under physiological conditions. Nature. 391:169-173.

8. Stamler, J.S., et al. 1997. Blood flow regulation by S-nitrosohemoglobin in the physiological oxygen gradient. Science. 276:2034-2037.

9. Head, C.A., et al. 1997. Low concentrations of nitric oxide increase oxygen affinity of sickle erythrocytes in vitro and in vivo. J. Clin. Invest. 100:1193-1198.

10. Guarnone, R., Centenara, E., and Barosi, G. 1995. Performance characteristics of Hemox-Analyzer for assessment of the hemoglobin dissociation curve. Haematologica. 80:426-430.

11. Green, L.C., et al. 1982. Analysis of nitrate, nitrite, and [15N]nitrate in biological fluids. Anal. Biochem. 126:131-138.

12. Samouilov, A., and Zweier, J.L. 1998. Development of chemiluminescence-based methods for specific quantitation of nitrosylated thiols. Anal. Biochem. 258:322-330.

13. McMahon, T.J., and Stamler, J.S. 1999. Concerted nitric oxide/oxygen delivery by hemoglobin. Methods Enzymol. 301:99-114.

14. Winterbourn, C.C., and Carrell, R.W. 1977. Oxidation of human haemoglobin by copper. Mechanism and suggested role of the thiol group of residue beta-93. Biochem. J. 165:141-148.

15. Patel, R.P., et al. 1999. Biochemical characterization of human S-nitrosohemoglobin. Effects on oxygen binding and transnitrosation. J. Biol. Chem. 274:15487-15492.

16. Garel, M.C., Domenget, C., Galacteros, F., Martin-Caburi, J., and Beuzard, Y. 1984. Inhibition of erythrocyte sickling by thiol reagents. Mol. Pharmacol. 26:559-565.

17. Garel, M.C., et al. 1986. Covalent binding of glutathione to hemoglobin. I. Inhibition of hemoglobin S polymerization. J. Biol. Chem. 261:14704-14709.

18. Pawloski, J.R., Swaminathan, R.V., and Stamler, J.S. 1998. Cell-free and erythrocytic S-nitrosohemoglobin inhibits human platelet aggregation. Circulation. 97:263-267.

19. Fox-Robichaud, A., et al. 1998. Inhaled NO as a viable antiadhesive therapy for ischemia/reperfusion injury of distal microvascular beds. J. Clin. Invest. 101:2497-2505.

20. Gladwin, M.T., Schechter, A.N., Shelhamer, J.H., and Ognibene, F.P. 1999. The acute chest syndrome in sickle cell disease. Possible role of nitric oxide in its pathophysiology and treatment. Am. J. Respir. Crit. Care Med. 159:1368-1376.

21. Atz, A.M., and Wessel, D.L. 1997. Inhaled nitric oxide in sickle cell disease with acute chest syndrome. Anesthesiology. 87:988-990.

22. French, J.A., II, et al. 1997. Mechanisms of stroke in sickle cell disease: sickle erythrocytes decrease cerebral blood flow in rats after nitric oxide synthase inhibition. Blood. 89:4591-4599.

23. Funai, E.F., Davidson, A., Seligman, S.P., and Finlay, T.H. 1997. Snitrosohemoglobin in the fetal circulation may represent a cycle for blood pressure regulation. Biochem. Biophys. Res. Commun. 239:875-877.

24. Takahashi, Y., et al. 1998. Nitrosyl hemoglobin in blood of normoxic and hypoxic sheep during nitric oxide inhalation. Am. J. Physiol. 274:H349-H357.

25. Rodgers, G.P., et al. 1990. Microcirculatory adaptations in sickle cell anemia: reactive hyperemia response. Am. J. Physiol. 258:H113-H120.

26. Lonsdorfer, J., et al. 1983. Cardiorespiratory adjustments in chronic sickle cell anemia. Bull. Eur. Physiopathol. Respir. 19:339-344. 